\title{
Cardiac Valvular Regurgitation Severity
}

National Cancer Institute

\section{Source}

National Cancer Institute. Cardiac Valvular Regurgitation Severity. NCI Thesaurus. Code C102244.

The qualitative measurement of the severity of cardiac valvular regurg itation. 